\title{
Phospholipid Scramblase 1 Functionally Interacts with Angiogenin and Regulates Angiogenin-Enhanced rRNA Transcription
}

\author{
Junqiao Zhu ${ }^{\mathrm{a}}$ Jinghao Sheng ${ }^{\mathrm{a}}$ Haojie Dong ${ }^{\mathrm{a}}$ Lan Kang $^{\mathrm{b}}$ Jian Ang \\ Zhengping $\mathrm{Xu}^{\mathrm{a}}$
}

anstitute of Environmental Medicine, Zhejiang University School of Medicine, 'Intensive Care Unit, the First Affiliated Hospital of Zhejiang University School of Medicine, 'Department of Gastroenterology, the Second Affiliated Hospital of Zhejiang University School of Medicine, Hangzhou, China

\section{Key Words}

Angiogenin - Phospholipid scramblase 1 - Ribosome RNA transcription - Protein-protein interaction

\begin{abstract}
Background: Angiogenin (ANG) can translocate to the target cell nucleus and accumulate in the nucleolus to enhance rRNA transcription, thus promoting cell proliferation. However, the regulation of ANG-enhanced rRNA transcription remains unknown. Previously we identified phospholipid scramblase 1 (PLSCR1) as a potential ANG-interacting protein in yeast two-hybrid screening. Methods: The interaction was re-confirmed in yeast cells and further verified by in vitro pull down, in vivo co-immunoprecipitation (Co-IP), fluorescent resonance energy transfer (FRET) and immunofluorescence analyses. The rRNA transcription level was determined by real-time quantitative PCR and Northern blot. Results: PLSCR1 was identified as a novel ANGinteracting protein. Notably, PLSCR1 interacted with ANG in the cell nucleus and regulated rRNA transcription. Furthermore, depletion of cellular ANG expression abolished PLSCR1enhanced rRNA transcription, which could be rescued by exogenous ANG. Conclusion: Our data suggest that PLSCR1 positively regulates rRNA transcription through interacting with ANG, thus deepening our understanding on rRNA transcription regulation.
\end{abstract}




\section{Introduction}

Angiogenin (ANG) is a 14-kDa protein originally isolated from the conditioned medium of HT-29 human colon adenocarcinoma cells [1]. It exerts its angiogenic function by activating vessel endothelial cells and promoting a series of cellular processes, including cell migration, invasion, proliferation, and formation of tubular structures [2, 3]. Besides its angiogenic function, ANG could directly stimulate cancer cell proliferation [4, 5]. Recently, ANG has been found to be expressed in motor neurons, and its loss of function mutations are associated with amyotrophic lateral sclerosis and Parkinson's disease [6-10], indicating a neuroprotective role of this protein. A number of cell types have so far been reported as ANG's targeting cells, such as vessel endothelial and smooth muscle cells, tumor cells, and motor neurons $[3,5,7]$.

Accumulating evidences indicate that the function of ANG on cell growth and survival is related with its nuclear activity in enhancing rRNA transcription [11]. ANG could translocate to the nucleus of its target cells where it accumulates in the nucleolus, binds to the promoter region of ribosomal DNA (rDNA), and stimulates rRNA transcription. It has been reported that ANG-mediated rRNA synthesis is critical for cell proliferation and the maintenance of normal physiological function of motor neurons [11, 12]. However, the molecular mechanisms underlying the ANG-enhanced rRNA transcription and its regulation remain elusive.

Protein-protein interactions play key roles in essential every biological process. To looking for protein partners that may involve in ANG-enhanced rRNA transcription, we performed a yeast two-hybrid screen using ANG as bait, and identified phospholipid scramblase 1 (PLSCR1) as a potential ANG binding protein. In this study, we confirmed the authenticity of the interaction between PLSCR1 and ANG both in vitro and in vivo. Our further study revealed that the interaction happened in the nucleus of HeLa cells and positively regulated rRNA transcription.

\section{Materials and Methods}

\section{Plasmid construction}

ANG cloning and pDBLeu-ANG bait generation for yeast two-hybrid analysis were described previously [13]. The obtained prey, pEXPAD502-PLSCR1, was used as the origin of PLSCR1 gene. The full-length PLSCR1 was cloned into the Eco RI/Not I sites of pET-42a-c(+) (Novagen, Darmstadt, Germany) to produce pETPLSCR1 for expressing GST-fused protein and subsequently used for the GST-pull-down assay. To generate a construct for hemagglutinin (HA)-tagged protein expression, the PLSCR1 gene was subcloned into the Sal I/Not I sites of pCI-neo mammalian expression vector (Promega, Madison, WI, USA) which had been preinserted a $3 \times \mathrm{HA}$ tag into the Eco RI/Xba I sites. For fluorescent resonance energy transfer (FRET) assay, the plasmids pYFP-ANG and pCFP-PLSCR1 were constructed to express yellow fluorescent protein (YFP) fused ANG and cyan fluorescent protein (CFP) tagged PLSCR1 respectively following the same procedure as described in [14]. All the constructs were sequenced to verify their reading frames.

The ANG RNA interference (RNAi) cassette containing plasmid pAng-RNAi, the empty vector pBS/U6 and pBabe-puro were described previously [15]. The siRNA for PLSCR1 interference was synthesized by the GenePharma Co. (Shanghai, China) with a target sequence 5'-AGT CTC CTC AGG AAA TCT G-3' according to the original report [16].

\section{Yeast two-hybrid assay}

The PROQUEST ${ }^{\mathrm{TM}}$ Two-Hybrid System (Invitrogen, Grand Island, NY, USA) was used to confirm the interaction between ANG and PLSCR1 according to the manufacturer's instruction. Briefly, S.cerevisiae strain MaV203 was co-transformed with the bait plasmid pDBLeu-ANG and the prey plasmind pEXPAD502PLSCR1, and selected on selective drop-out (SD) plates lacking histidine, leucine and tryptophan (SD-HisLeu-Trp). 3-Aminotriazole ( $50 \mathrm{mM}$ ) was added to inhibit HIS3 leakiness. After incubated at $30{ }^{\circ} \mathrm{C}$ for 7 days, the positive colonies were subjected to the X-gal (5-bromo-4-chloro-3-indolyl-beta-D-galactopyranoside) 
assay. Yeast control strains (a-e), which represent none interaction (a), weak interaction (b), moderately strong interaction (c), strong interaction (d), and very strong interaction (e) respectively, were applied to the X-gal analysis simultaneously (Fig. 1A). Clones showing blue color within $12 \mathrm{~h}$ were designated as positive clones.

\section{Western blotting}

The protein samples were resolved by $12 \%$ SDS-PAGE, and then transferred to a PVDF (Millipore, Billerica, MA, USA) by semi-dry transfer in Towbin's buffer (20\% methanol, 25 mMTris, $192 \mathrm{mM}$ glycine), blocked with 5\% skim milk powder in Tris-buffered saline with 0.1\% Tween-20 (TBST). All primary and secondary antibodies were diluted in $5 \%$ skim milk powder in TBST. The primary antibodies included antiANG polyclonalantibody (home-made), anti-PLSCR1 monoclonal antibody IE9, anti-fibrillarin monoclonal antibody $\mathrm{C} 13 \mathrm{C} 3$, anti-HA monoclonal antibody 6E2, anti-beta-tubulin polyclonal antibody $9 \mathrm{~F} 3$ and antialpha-tubulin monoclonal antibody 11H10 (Cell Signaling Technology, Danvers, MA, USA). Detected proteins were visualized using enhanced chemiluminescence (Pierce, Rockford, IL, USA).

\section{GST-pull-down assay}

The pET-PLSCR1 was transformed into E. coli BL21 (DE3) for bacterial expression of the GST chimeric protein. The bacteria were induced with $0.5 \mathrm{mM}$ isopropyl-1-thio-b-D-galactopyranoside (IPTG) for $12 \mathrm{~h}$ at $20^{\circ} \mathrm{C}$, harvested and then sonicated in PBS buffer containing a protease inhibitor cocktail (Roche Applied Science, Indianapolis, IN, USA) and DTT (5 mM). Lysate containing GST or GST-PLSCR1 fusion protein was pre-cleared by ultra-centrifugation and batch-bound overnight to GSH-agarose (Amersham Biosciences, Pittsburgh, PA, USA). Then the beads containing about $0.1 \mu \mathrm{g}$ of GST or GST-PLSCR1 chimeric protein were incubated with $0.5 \mu$ g of ANG in a binding buffer ( 20 mMTris, $250 \mathrm{mMNaCl}, 1 \mathrm{mM}$ EDTA, $0.5 \%$ NP-40) at 4 ${ }^{\circ} \mathrm{C}$ for $6 \mathrm{~h}$. The beads were washed, and the bead-bound proteins were subjected to SDS-PAGE and Western blotting.

\section{Cell culture, transfection and nuclear translocation of ANG}

HeLa cells were maintained in Dulbecco's modified Eagle's medium (DMEM) supplemented with $10 \%$ fetal bovine serum (FBS). Cells were transfected at 50-60\% confluency using the lipofectamine ${ }^{\mathrm{TM}} 2000$ reagent (Invitrogen) in accordance with the manufacturer's protocol. ANG stable knocking-down HeLa cells were generated according to Tsuji's protocol [5], and the interference efficiency was determined in both mRNA and protein levels by using quantitative RT-PCR and immunoblotting approaches respectively. For nuclear translocation of exogeneous ANG, HeLa cells were incubated with $0.5 \mu \mathrm{g} / \mathrm{ml}$ of ANG at $37{ }^{\circ} \mathrm{C}$ and monitored by indirect immunofluorescence assay.

\section{Immunofluorescence assay}

To visualize the nuclear localizations of ANG and/or PLSCR1, HeLa cells were grown on glass coverslips and fixed with pre-chilled methanol $\left(10 \mathrm{~min},-20^{\circ} \mathrm{C}\right)$. After blocking for $1 \mathrm{~h}$ in $1 \% \mathrm{BSA}$, the fixed cells were incubated with $30 \mu \mathrm{g} / \mathrm{mL}$ of anti-ANG monoclonal antibody 26-2F (provided by Dr. Guo-fu Hu at Tufts University) and/or anti-PLSCR1 polyclonal antibody (Abnova, Taipei, China) with 1:100 dilution for $1 \mathrm{~h}$, washed, and incubated with FITC-conjugated goat anti-mouse and TRITC-conjugated goat anti-rabbit secondary antibody at 1:100 dilution for $1 \mathrm{~h}$. Anti-fibrillarin antibody was used to stain the nucleolus. Slides were mounted in mounting solution (50\% glycerol) and observed under a confocal microscope (Zeiss, Hamburg, Germany).

\section{In vivo co-immunoprecipitation assay}

The total cell lysate was extracted with lysis buffer (1\% Triton X-100, $100 \mathrm{mM} \mathrm{NaCl}, 100 \mathrm{mM}$ Tris pH 7.5, $0.5 \mathrm{mM}$ EDTA, 10\% glycerol, and protease inhibitor cocktail), and cleared by centrifugation at $16,000 \mathrm{~g}$ for $30 \mathrm{~min}$ at $4{ }^{\circ} \mathrm{C}$. Protein concentration was determined using a modified Bradford protocol.

To obtain the nuclear lysate preparation, the cells were washed twice with ice-cold PBS and resuspended in Chelsky Buffer $(10 \mathrm{mM}$ Tris- $\mathrm{HCl}, 10 \mathrm{mM} \mathrm{NaCl}, 3 \mathrm{mM} \mathrm{MgCl}, 30 \mathrm{mM}$ Sucrose, and 1 mMphenylmethylsulfonyl fluoride (PMSF)) containing complete protease inhibitor mixture (Roche Applied Science). The suspension was incubated on ice for 10 min before adding $10 \%$ Triton-X-100 to a final concentration of $0.3 \%$ to allow a sufficient breakage of the cytoplasmic membrane. After centrifugation at 
$1,200 \mathrm{~g}$ for $5 \mathrm{~min}$ at $4{ }^{\circ} \mathrm{C}$, the pellet was obtained and lysed in lysis buffer (100 mM Tris- $\mathrm{HCl}$, pH 7.5, $100 \mathrm{mM}$ $\mathrm{NaCl}, 20 \mathrm{mM} \mathrm{MgCl}_{2}, 1 \%$ Triton-X-100, and $1 \mathrm{mM}$ PMSF) containing complete protease inhibitor mixture, then centrifuged again at $16,000 \mathrm{~g}$ for $30 \mathrm{~min}$ at $4{ }^{\circ} \mathrm{C}$.

Equal amounts of the resultant supernatant were incubated overnight with the goat original antiANG polyclonal antibody or control IgG $\left(4^{\circ} \mathrm{C}\right.$ on rotator), followed by incubation with Protein G-conjugated agarose beads (Millipore). The immune-precipitates were analyzed by Western blotting.

Confocal microscopy and fluorescent resonance energy transfer (FRET) analysis

HeLa cells were co-transfected with pYFP-ANG and pCFP-PLSCR1 plasmids and the fluorescence was examined by confocal microscope with a $40 \times$ oil immersion objective. Confocal microscopic images were obtained with a Leica confocal imaging spectrophotometer system (TCS-SP). Photobleaching based FRET assay was performed as previously described [14]. Briefly, the fluorescence images for CFP and YFP were collected repetitively at a 10 seconds interval for five times. After that, YFP was photobleached with the full power of the $514 \mathrm{~nm}$ line laser, and the emission intensities of CFP and YFP were monitored for an additional five times. FRET efficiency (FRETe) was calculated as the percentage of the CFP fluorescence recovery $\left[\mathrm{FRETe}=\left(\mathrm{CFP}_{\mathrm{A}}-\mathrm{CFP}_{\mathrm{B}}\right) / \mathrm{CFP}_{\mathrm{A}} \times 100 \%\right]$, where $\mathrm{CFP}_{\mathrm{A}}$ was the CFP intensity after photobleaching and $\mathrm{CFP}_{\mathrm{B}}$ was the CFP intensity before photobleaching of YFP.

\section{Quantitative real-timePCR}

Total RNA was extracted using a TRIzoL Reagent (Invitrogen). For each reverse transcription, $2 \mu \mathrm{g}$ of total RNA was converted into cDNA by moloney murine leukemia virus reverse transcriptase (Promega). An equal volume mixture of the products was then quantified by quantitative real-time PCR using an ABI PRISM 7500 Sequence Detector and Power SYBR Green PCR Master Mix (Applied Biosystems, NY, USA). PCR was done with an initial denaturation step of $10 \mathrm{~min}$ at $95^{\circ} \mathrm{C}$ followed by 40 cycles of amplification consisting of $15 \mathrm{~s}$ at $95^{\circ} \mathrm{C}, 15 \mathrm{~s}$ at $59^{\circ} \mathrm{C}$, and $35 \mathrm{~s}$ at $72{ }^{\circ} \mathrm{C}$. The primers for $47 \mathrm{~S}$ pre-rRNA were $5^{\prime}$-CCT GCT GTT CTC TCG CGC GTC CGA G-3' and 5'-GCG TCT CGT CTC GTC TCA CT-3'. The primers for ANG were 5'-CAG GAT AAC TCC AGG TAC-3' and 5'-CGG ACG ACG GAA AAT TG-3'. The primers for PLSCR1 were 5'-AGA ACA GCT TTG GAC AGA GGG TTT ACT TTG C-3' and 5'-CTG AAG GCA GCA GGG ACA ACA ACA G-3'. The primers for the internal control gene GAPDH were 5'-ACG GAT TTG GTC GTA TTG GG-3' and 5'-CGC TCC TGG AAG ATG GTG AT-3'. Each sample was run in triplicate. Average threshold cycle (Ct) values for PLSCR1, ANG or 47S pre-rRNA was normalized against the average $\mathrm{Ct}$ values for $G A P D H$ from the same cDNA sample. Fold change between experimental groups (E) and control groups (C) equals $2^{-\Delta \Delta \mathrm{Ct}}$, where $\Delta \mathrm{Ct}=\mathrm{Ct}_{\mathrm{PLSCR} 1 / \mathrm{ANG} / 45 \mathrm{~S}}-\mathrm{Ct}_{\mathrm{GAPDH}}$, and $\Delta \Delta \mathrm{Ct}=$ $\Delta \mathrm{Ct}_{\mathrm{E}}-\Delta \mathrm{Ct}_{\mathrm{C}}$.

\section{Northern Blot}

$10 \mu \mathrm{g}$ total RNA from each groups were separated by electrophoresis through a $1 \%$ agarose/2\% formaldehyde gel, and transferred overnight in $20 \times$ saline-sodium citrate (SSC) to a positively charged nylon membrane. Northern blots were performed with Digoxin (DIG)-labeled DNA probes specific for 47S pre-rRNA or $\beta$-actin mRNA. The probe used for 47 s pre-rRNA is $5^{\prime}$ - GGT CGC CAG AGG ACA GCG TGT CAG (DIG)-3' that hybridizes with the first 25 nucleotides of the 47s rRNA precursor [15]. The probe used for $\beta$-actin is 5'-AGG GAT AGC ACA GCC TGG ATA GCA AC (DIG)-3' which hybridizes with the $\beta$-actin mRNA at 484 to 509. Blots were detected using DIG Northern Starter Kit from Roche Applied Science following the manufacturer's instructions.

Statistical analysis

Data were presented as means \pm S.D. The differences between groups were determined by Student's t-test (two-tailed). Differences were declared significant at *: $P<0.05$ and ${ }^{* *}: P<0.01$.

\section{Results}

PLSCR1 binds to ANG in vitro and in vivo

Since PLSCR1 was picked as a potential ANG-interacting protein from yeast two-hybrid screening, we first re-confirmed the interaction in the yeast cells. For this purpose, yeast cells 
A.
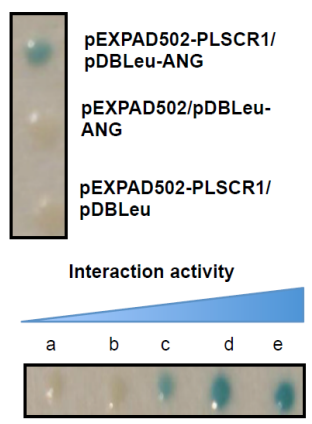

D.
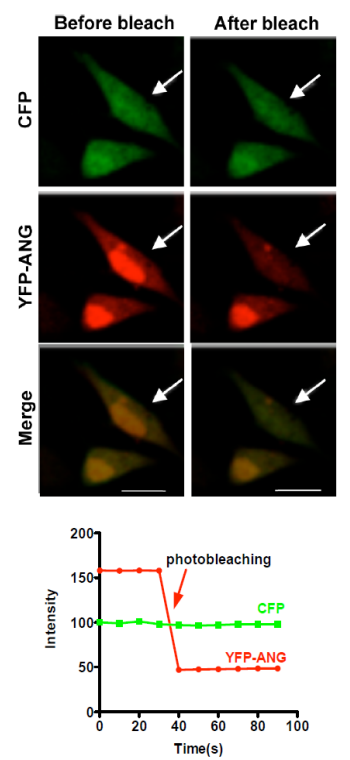

B.

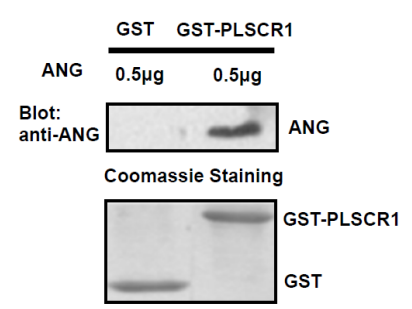

c.

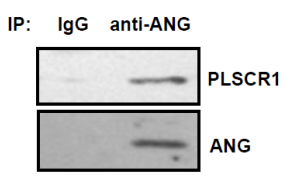

$5 \%$ Input

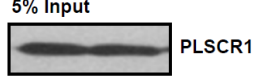

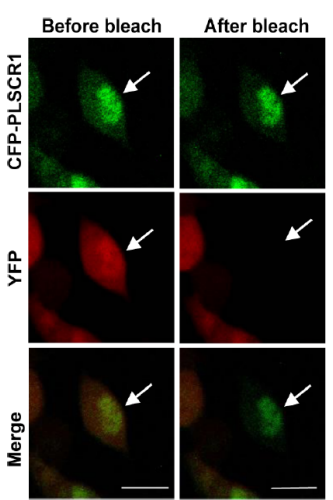
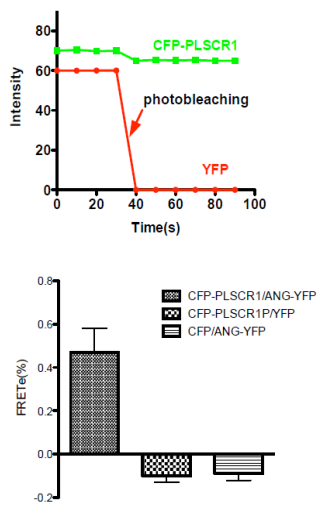
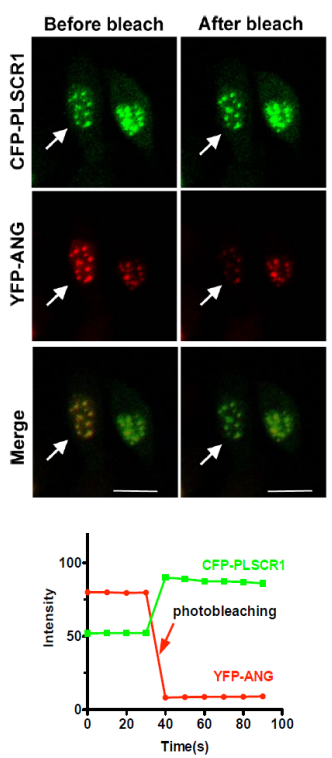

Fig. 1. PLSCR1 interacts with ANG in vitro and in vivo. A. Yeast strain MaV203 cells were co-transformed with pEXP-AD502 or pEXP-AD502-PLSCR1 along with pDBLeu or pDBLeu-ANG as indicated. Transformants and yeast control strains (a-e) were subjected to X-gal analysis. The $\beta$-galactosidase-positive colony (blue staining) indicates a positive interaction, while white colonies indicate no interaction. B. $0.5 \mu \mathrm{g}$ recombinant ANG was incubated with recombinant GST-tagged PLSCR1 in the presence of GSH-agarose beads. The pulldown products were examined by immunoblotting with anti-ANG polyclonal antibody (top panel) and coomassie blue staining (bottom panel). C. PLSCR1 was co-immunoprecipitated with ANG in HeLa cells. The cell lysates were incubated with anti-ANG antibody, or normal IgG as control, and the immunoprecipitates were subjected to immunoblot by anti-ANG and anti-PLSCR1 antibodies respectively. The input represents $5 \%$ of the lysate used in each binding assay (bottom panel). D. HeLa cells were co-transfected with plasmids as indicated and subjected to photobleaching analysis. The bleached cell was marked with a white arrow. The CFP and YFP fluorescence emission intensities in the cells before and after photobleaching were shown in right panel. The FRET efficiency (FRETe) was calculated as the percentage of the CFP fluorescence recovery. All the data represented the means of three independent experiments with the bars showing standard deviation. Bar scale: $20 \mu \mathrm{m}$. 
were co-transformed with pDBLeu or pDBLeu-ANG along with pEXPAD502 or pEXPAD502PLSCR1. The results showed that the transformants turned blue in X-gal analysis only when ANG and PLSCR1 were simultaneously expressed (Fig. 1A). Comparing to the yeast control strains, the interaction between ANG and PLSCR1 was moderately strong in this system (Fig. 1A).

To further verify the interaction, we performed in vitro GST pull-down and in vivo coimmunoprecipitation assay. When ANG was co-incubated with bacteria-expressed and beadbound GST-PLSCR1 or GST, it could be specifically pulled down by GST-PLSCR1 but not by GST (Fig. 1B), indicating a directly physical interaction between ANG and PLSCR1 in vitro. We then ectopically expressed HA-tagged PLSCR1 in HeLa cells, and applied $0.5 \mu \mathrm{g} / \mathrm{ml}$ ANG into the culture medium for $1 \mathrm{~h}$. After washing, the cells were harvested, lysed and subjected to co-immunoprecipitation with an anti-ANG antibody. The subsequent immunoblotting analysis revealed that PLSCR1 was precipitated together with ANG (Fig. 1C), indicating that PLSCR1 interacts with ANG in the cellular content.

We then applied FRET technique to test PLSCR1-ANG interaction. After co-transfecting HeLa cells with pCFP-PLSCR1 and pYFP-ANG, the energy acceptor was photobleached by a high-intensity exposure to the YFP excitation light (514 nm laser) to block energy transfer from CFP to YFP, and the energy redistribution of CFP was recorded. If there is a FRET, photobleaching of YFP should increase the emission of CFP at $480 \mathrm{~nm}$. As shown in the Figure 1D, the FRET did occur between PLSCR1 and ANG, further confirming that PLSCR1 interacts with ANG in living cells. The FRETe of the two control groups CFP/YFP-ANG and CFP-PLSCR1/YFP were $-10 \pm 3 \%$ and $-9 \pm 3 \%$ respectively, while the FRETe of CFP-PLSCR1/ YFP-ANG was $47 \pm 11 \%$ (Fig. 1D, Bar graph).

Interestingly, co-expression of CFP-PLSCR1 and YFP-ANG changed both proteins' localization and formed clear dots in the nucleus (Fig. 1D), suggesting that PLSCR1 and ANG interact with each other in cell nucleus.

PLSCR1 binds to ANG in the nuclei of HeLa cells

We then examined the interaction between endogenous ANG and PLSCR1 in Hela cells. Immunofluorescence results revealed that ANG was predominantly localized in the nucleus while the PLSCR1 was distributed both in nucleus and cytoplasm membrane (Fig. 2A). Merged image showed that endogenous ANG and PLSCR1 were co-localized in the nucleus of HeLa cells, especially in the nucleolus (Fig. 2A), suggesting that the interaction between ANG and PLSCR1 might happen in the nucleolus. Thereafter, we analyzed the localization of ANG and PLSCR1 by subcellular fractionation. The data revealed that ANG was predominantly found in the nuclear fraction while PLSCR1 was present in both nuclear and cytoplasmic fractions (Fig. 2B). To further confirm that ANG interacts with PLSCR1 in the nucleus, we took the nuclear fraction to do nuclear co-immunoprecipitation assay. As expected, we were able to co-immunoprecipitate PLSCR1 with an ANG specific polyclonal antibody, but not the normal IgG (Fig. 2C), indicating that endogenous PLSCR1 interacts with ANG in the nuclei of HeLa cells.

\section{PLSCR1 positively regulates rRNA transcription in HeLa cells}

The finding that PLSCR1 interacted with ANG in the nucleus prompted us to examine whether PLSCR1 is involved in the ANG-stimulated rRNA transcription. We first manipulated the PLSCR1 expression in HeLa cells by transfecting a PLSCR1 expressing plasmid or smallinterfering RNAs (siRNAs), and then measured the 47S pre-rRNA level by RT-qPCR and Northern blot to evaluate the rRNA transcription activity. The RT-qPCR results showed that over-expression of PLSCR1 led to about 2.5-fold increase of steady-state 47S pre-rRNA level in HeLa cells compared with control (Fig. 3A and B). Knocking down the endogenous PLSCR1 expression by siRNA resulted in about $40 \%$ decrease in $47 \mathrm{~S}$ rRNA compared with its negative control (Fig. 3C and D). The Northern blot also showed similar results (Fig. 3E). These data demonstrate that PLSCR1 is a positive regulator of rRNA transcription in HeLa cells. 
A.
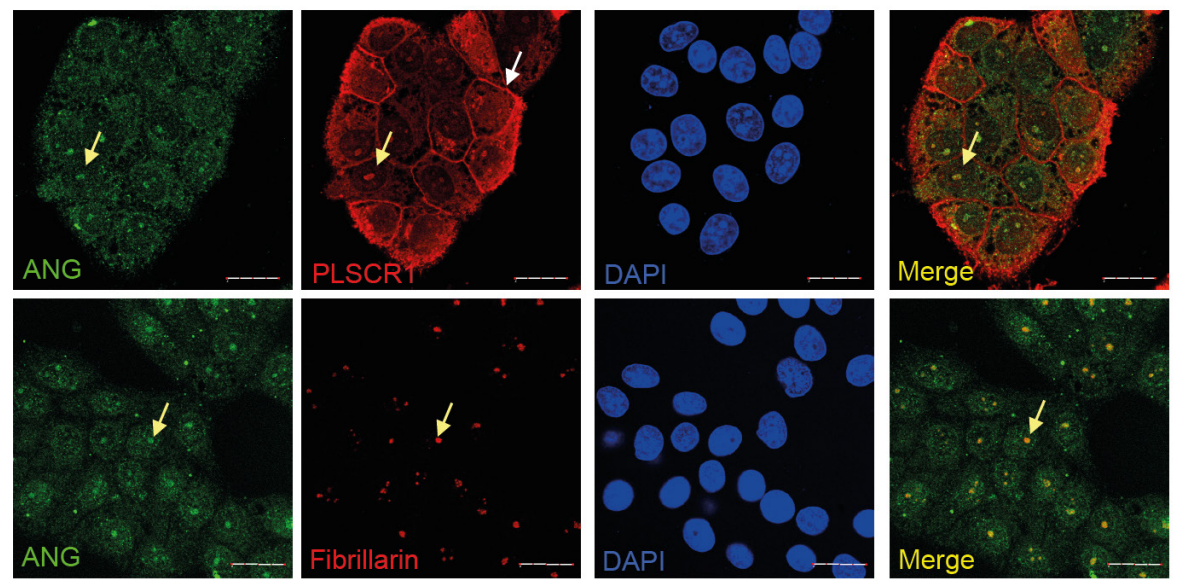

B.

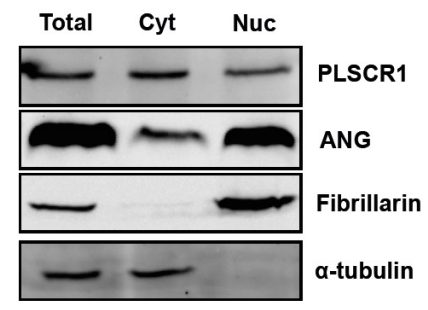

C.

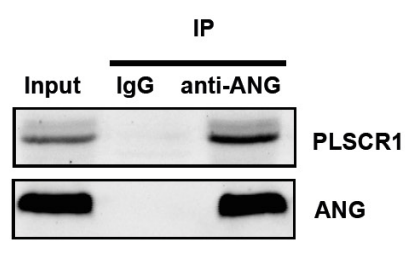

Fig. 2. PLSCR1 interacts with ANG in nucleus of HeLa cells. A. Subcellular localization of endogenous PLSCR1 and ANG. Non-transfected HeLa cells were subjected to immunofluorescence staining with anti-PLSCR1 and anti-ANG antibodies. Fibrillarin was stained to show the nucleolus. The yellow arrow indicates the nucleolus localization of ANG and PLSCR1. Part of the endogenous PLSCR1 also localized in the cell membrane (white arrow). Bar scale: $20 \mu \mathrm{m}$. B. PLSCR1 and ANG existed in cell nuclear fraction. Nuclear and cytoplasmic fractions were prepared from the non-transfected HeLa cells. Samples from equivalent numbers of cells were analyzed by immunoblotting with antibodies against PLSCR1, ANG, fibrillarin and $\alpha$-tubulin. Total, whole cell lysate; Cyt, cytoplasmic lysate; Nuc, nuclear lysate. C. Co-immunoprecipitation of nuclear PLSCR1 with ANG. Equivalent nuclear extracts prepared in (B) were subjected to co-immunoprecipitation with the anti-ANG polyclonal antibody or IgG, followed by immunoblotting with anti-PLSCR1 and anti-ANG antibodies respectively.

The regulation capacity of PLSCR1 on rRNA transcription depends on the availability of $A N G$

We next examined if PLSCR1-enhanced rRNA transcription depends on the existence of ANG. The ANG knockdown stable cells and control cells were established by our lab and have been described in previous published report [17]. To visually show the decreased expression of ANG in the cells, we examined the cellular ANG level by immunofluorescent staining. The pictures clearly revealed a much lower cellular ANG protein present in the cytoplasm and no detectable accumulation of this protein in the nucleoli upon ANG knockdown (Fig. 4A, a and b), which could be restored by adding exogenous ANG into the culture medium (Fig. 4A c).

We thus measured the cellular rRNA transcription activity in this ANG-down-regulated cell line with RT-qPCR. In accordance with the original report [5], knocking-down of ANG downregulated rRNA transcription and addition of exogenous ANG rescued rRNA transcription to control level (Fig. 4B control column). However, over-expression of PLSCR1 did not upregulate the $47 \mathrm{~S}$ rRNA level if the endogenous ANG was down-regulated, indicating that the ANG is required for PLSCR1-enhanced rRNA transcription (Fig. 4B PLSCR1 column). In the 
Fig. 3. PLSCR1 enhances rRNA transcription. A. Overexpression of PLSCR1 in HeLa cells. HeLa cells were transiently transfected with pCI-3HA vector (control) or pCI-3HAPLSCR1 plasmid (PLSCR1). Twenty-four hours after transfection, the cell lysate was extracted and the PLSCR1 expression level was determined by western blotting. $\beta$-tubulin was served as a loading control. B. PLSCR1 positively regulated pre-rRNA transcription in HeLa cells. The 47S pre-rRNA expression level in either control or PLSCR1 over-expressed cells was determined by RT-qPCR. C. siRNA against PLSCR1 efficiently knocked down the expression of endogenous PLSCR1. The level of endogenous PLSCR1 protein level was detected by immunoblotting. D. Kno-
A.

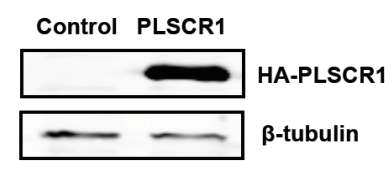

c.

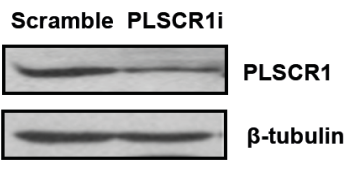

E.
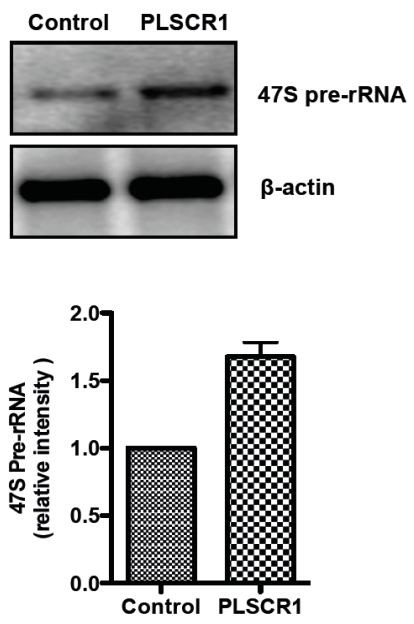

B.

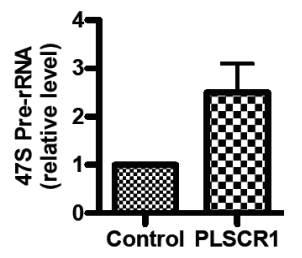

D.

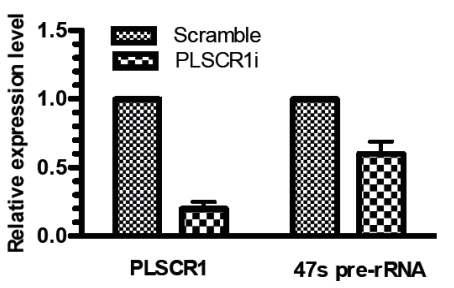

F.
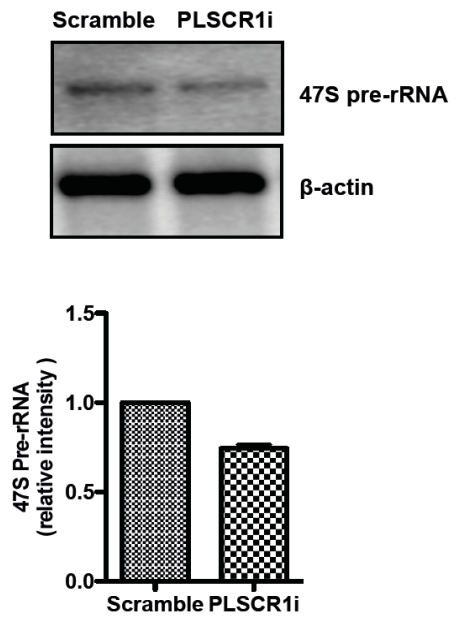

cking down of PLSCR1 decreased the rRNA transcription level in HeLa cells. Total RNA was extracted and the 47S pre-rRNA transcription level was determined by RT-qPCR. The GAPDH level was used as the internal control of RT-qPCR. E/F. Northern blot confirmed the enhancement of PLSCR1 on rRNA transcription. Top panel, Northern blots were detected with DIG-labeled probes specific to the 47S pre-rRNA and $\beta$-actin mRNA. Bottom panel, quantification of the $47 \mathrm{~S}$ pre-rRNA relative to $\beta$-actin mRNA by ImageJ analysis.

rescue experiment, introduction of exogenous ANG could recover the PLSCR1-enhanced rRNA transcription, indicating that the regulation of PLSCR1 on rRNA transcription is ANG dependent (Fig. 4B). The results were also confirmed by the Northern blot assay (Fig. 4C). These findings suggest that the interaction between ANG and PLSCR1 plays an important role in regulating rRNA transcription.

\section{Discussion}

ANG-stimulated rRNA transcription plays a critical role in vessel endothelial cell proliferation, cancer cell proliferation, and motor neuron survival [11, 12]. However, the mechanism of how this process is regulated remains unknown. In this study, we demonstrated 
Fig. 4. The regulation capacity of PLSCR1 on rRNA transcription depends on the availability of ANG. A. HeLa cells were transfected with pAng-RNAi and its control vector $\mathrm{pBS}$ / U6. Stable transfectants were selected with $0.5 \mu \mathrm{g} / \mathrm{ml} \mathrm{pu-}$ romycin. The 0.5 $\mu \mathrm{g} / \mathrm{ml}$ recombinant ANG were added to the medium as a rescued group. ANG was detected with monoclonal antibody 26-2F. The pictures were obtained under the same scanning parameter setting of confocal microscopy. Bar scale: $20 \mu \mathrm{m}$. B. PLSCR1-mediated enhancement of pre-rRNA level was ANG-dependent. The stable trans-
A.

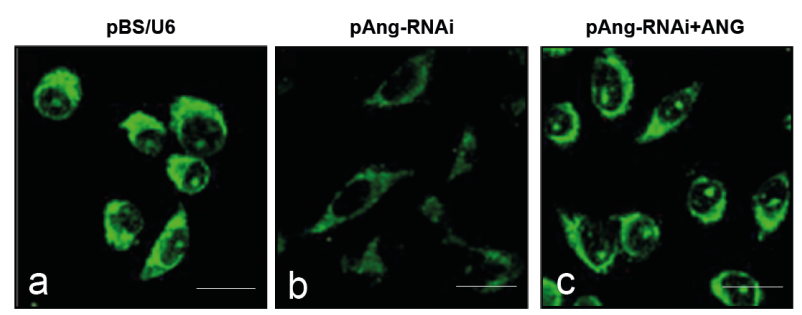

B.

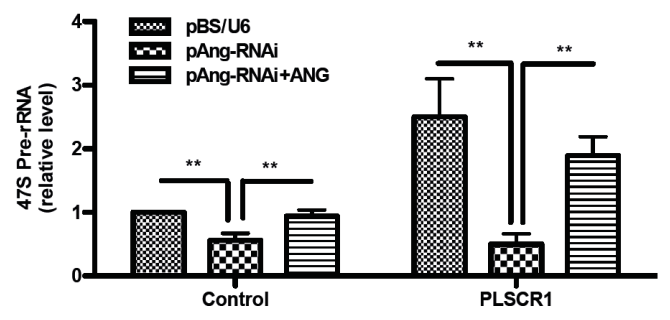

c.
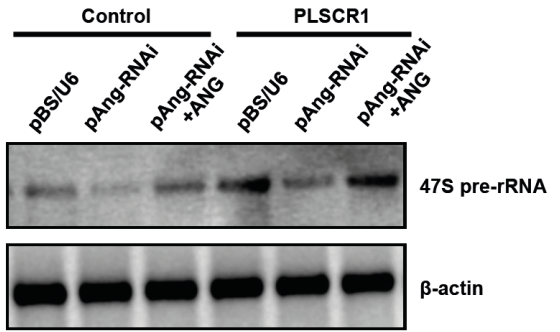

fectants of pBS/U6 and pAng-RNAi were transiently transfected with pCI-3HA vector or pCI-3HA-PLSCR1 plasmid. For the ANG rescue assay, exogenous ANG $(0.5 \mu \mathrm{g} / \mathrm{ml})$ was added back into the culture medium of the RNAi transfectant. 47S pre-rRNA transcription level was determined by RT-qPCR. The GAPDH level was used as the internal control of RT-qPCR. All the data represented the means of three independent experiments, with the bars showing standard deviation. ${ }^{* *}: P<0.01$. C. The $47 \mathrm{~S}$ pre-rRNA transcription level was analyzed by Northern blot. Left panel, Northern blots were detected with DIG-labeled probes specific to the $47 \mathrm{~S}$ pre-rRNA and $\beta$-actin mRNA. Right panel, quantification of the $47 \mathrm{~S}$ pre-rRNA relative to $\beta$-actin mRNA by ImageJ analysis.

that PLSCR1 interacts with ANG in the nucleus and up-regulates rRNA transcription in an ANG-dependent manner, suggesting that PLSCR1 regulates rRNA transcription through interacting with ANG. Therefore, this study provides evidence on how the ANG-stimulated rRNA transcription is further modulated by an ANG-interacting protein.

PLSCR1 was primarily identified as a multi-palmitoylated, calcium-binding endofacial membrane protein mediating nonselective and bidirectional transport of phospholipids between membrane leaflets $[18,19]$. Structural and functional analyses reveal that PLSCR1 has several functionally important domains, including multiple PXXP and PPXY domains, a DNA binding domain, a cysteine palmitoylation motif, a nonclassical nuclear localization signal (NLS), a $\mathrm{Ca}^{2+}$ binding EF-hand-like domain, and a single transmembrane domain [20], indicating its potential as a multifunction protein. Further studies do challenge its function 
as a major phospholipids translocator and reveal that PLSCR1 mainly acts as a signaling molecule and can translocate to nucleus [20].

Currently, little is known about the function of PLSCR1 in the nucleus. It has been reported that this protein binds to the promoter region $\left({ }^{-101} \mathrm{GTAACCATGTGGA}^{-89}\right)$ of the inositol 1,4,5-triphosphate receptor type 1 gene (IP3R1) and enhance its expression [21]. Here we reported that PLSCR1 promotes rRNA transcription although it's not clear whether this protein binds to genomic rDNA directly or indirectly. Interestingly, the binding site for PLSCR1 within IP3R1 promoter contains an E-box core consensus sequence (i.e.CANNTG), and one rRNA gene contains three CATGTG sequences with two in 18s and 28s coding regions and one in intergenic space, and 12 palindromic canonical sequence of CACGTG at various positions, suggesting PLSCR1 might directly bind to rDNA. Furthermore, PLSCR1 hosts a putative transcriptional activation domain [20]. Therefore, it would be worth to determine if PLSCR1 activates rRNA transcription through direct binding to these sequences. On the other hand, ANG has been reported to bind to ABE in the rRNA gene and stimulate rRNA transcription $[22,23]$. Therefore, it is also possible that PLSCR1 and ANG form a complex through their nuclear interaction to coordinate the transcriptional regulation capacities of different elements such as E-box and ABE.

To demonstrate that PLSCR1 regulates rRNA transcription through interacting with ANG, a possible method is to disrupt their intermolecular interaction by a competitive peptide mimicking the binding sequence and then observes the change of pre-rRNA transcription. Unfortunately, although we tried to map the binding sites on both proteins by yeast twohybrid approach, the results revealed that a broad range of peptides involved (data not shown), suggesting a possible conformational binding epitopes. Docking analysis predicted multiple binding regions between these two proteins (Data not shown). Interestingly, Kusano and Eizuru found a very similar binding pattern in PLSCR1 with its interaction partner. They reported that PLSCR1 directly interacts with the amino-terminal region of human T-cell leukemia virus type-1 (HTLV-1) Tax through its 1-100 and 200-250 amino acid regions [24]. Therefore, currently we could not provide direct evidence to prove that PLSCR1 interacts with ANG and then regulates ANG-stimulated rRNA transcription.

PLSCR1 has been implicated in growth factor signaling pathway regulation, cell proliferation, and tumor growth and development [20, 25-28]. However, its underlying mechanism of action remains unclear. Ribosome biogenesis is a fundamental step in cell growth and proliferation, and rRNA synthesis is critical to ribosome assembly. Therefore, our finding that PLSCR1 interacts with ANG in the cell nucleus and thus synergistically regulates rRNA transcription might provide one explanation for the mechanism. Intriguingly, ANG is reported as a permissive factor for proliferation role of growth factor EGF, bFGF, and VEGF [15], and our unpublished data reveal that these factors could increase PLSCR1 expression and nuclear translocation (data not shown), it is possible that the PLSCR1-ANG interaction as well as the up-regulation on rRNA transcription could be one of the down-stream and critical events in response to these growth factors. Taken together, our finding drives us to speculate that PLSCR1 may cooperate with ANG in promoting rRNA transcription, ribosome biogenesis, and cell proliferation.

Overall, the present study identifies nuclear PLSCR1 as an ANG-interacting protein and provides evidence that nuclear PLSCR1 regulates ANG-enhanced rRNA transcription. These data shed light on the nuclear role of PLSCR1 and the mechanism of rRNA transcription regulation.

\section{Acknowledgements}

We would like to thank Dr. Guo-fu Hu (Tufts University Medical Center, Boston, MA, U.S.A.) for the gift of ANG RNAi plasmid and the ANG-specific monoclonal antibody 26-2F. The work was supported by grants from National Natural Science Foundation of China (No. 30800173 and 31170721). 


\section{References}

1 Fett JW, Strydom DJ, Lobb RR, Alderman EM, Bethune JL, Riordan JF, Vallee BL: Isolation and characterization of angiogenin, an angiogenic protein from human carcinoma cells. Biochemistry 1985;24:5480-5486.

2 Riordan JF: Angiogenin. Methods Enzymol 2001;341:263-273.

- 3 Xu Z, Monti DM, Hu G: Angiogenin activates human umbilical artery smooth muscle cells. Biochem Biophys Res Commun 2001;285:909-914.

4 Yoshioka N, Wang L, Kishimoto K, Tsuji T, Hu GF: A therapeutic target for prostate cancer based on angiogenin-stimulated angiogenesis and cancer cell proliferation. Proc Natl Acad Sci U S A 2006;103:14519-14524.

-5 Tsuji T, Sun Y, Kishimoto K, Olson KA, Liu S, Hirukawa S, Hu GF: Angiogenin is translocated to the nucleus of hela cells and is involved in ribosomal rna transcription and cell proliferation. Cancer Res 2005;65:13521360 .

6 Greenway MJ, Andersen PM, Russ C, Ennis S, Cashman S, Donaghy C, Patterson V, Swingler R, Kieran D, Prehn J, Morrison KE, Green A, Acharya KR, Brown RH Jr, Hardiman O: Ang mutations segregate with familial and 'sporadic' amyotrophic lateral sclerosis. Nat Genet 2006;38:411-413.

7 Subramanian V, Crabtree B, Acharya KR: Human angiogenin is a neuroprotective factor and amyotrophic lateral sclerosis associated angiogenin variants affect neurite extension/pathfinding and survival of motor neurons. Hum Mol Genet 2008;17:130-149.

8 Subramanian V, Feng Y: A new role for angiogenin in neurite growth and pathfinding: Implications for amyotrophic lateral sclerosis. Hum Mol Genet 2007;16:1445-1453.

-9 Kieran D, Sebastia J, Greenway MJ, King MA, Connaughton D, Concannon CG, Fenner B, Hardiman O, Prehn JH: Control of motoneuron survival by angiogenin. J Neurosci 2008;28:14056-14061.

$\checkmark 10$ van Es MA, Schelhaas HJ, van Vught PW, Ticozzi N, Andersen PM, Groen EJ, Schulte C, Blauw HM, Koppers M, Diekstra FP, Fumoto K, LeClerc AL, Keagle P, Bloem BR, Scheffer H, van Nuenen BF, van Blitterswijk M, van Rheenen W, Wills AM, Lowe PP, Hu GF, Yu W, Kishikawa H, Wu D, Folkerth RD, Mariani C, Goldwurm S, Pezzoli G, Van Damme P, Lemmens R, Dahlberg C, Birve A, Fernandez-Santiago R, Waibel S, Klein C, Weber M, van der Kooi AJ, de Visser M, Verbaan D, van Hilten JJ, Heutink P, Hennekam EA, Cuppen E, Berg D, Brown RH Jr, Silani V, Gasser T, Ludolph AC, Robberecht W, Ophoff RA, Veldink JH, Pasterkamp RJ, de Bakker PI, Landers JE, van de Warrenburg BP, van den Berg LH: Angiogenin variants in parkinson disease and amyotrophic lateral sclerosis. Ann Neurol 2011;70:964-973.

11 Li S, Hu GF: Angiogenin-mediated rrna transcription in cancer and neurodegeneration. Int J Biochem Mol Biol 2010;1:26-35.

12 Li S, Hu GF: Emerging role of angiogenin in stress response and cell survival under adverse conditions. J Cell Physiol 2012;227:2822-2826.

$13 \mathrm{Hu}$ H, Gao X, Sun Y, Zhou J, Yang M, Xu Z: Alpha-actinin-2, a cytoskeletal protein, binds to angiogenin. Biochem Biophys Res Commun 2005;329:661-667.

14 Gao X, Hu H, Zhu J, Xu Z: Identification and characterization of follistatin as a novel angiogenin-binding protein. FEBS Lett 2007;581:5505-5510.

15 Kishimoto K, Liu S, Tsuji T, Olson KA, Hu GF: Endogenous angiogenin in endothelial cells is a general requirement for cell proliferation and angiogenesis. Oncogene 2005;24:445-456.

- 16 Zhao KW, Li X, Zhao Q, Huang Y, Li D, Peng ZG, Shen WZ, Zhao J, Zhou Q, Chen Z, Sims PJ, Wiedmer T, Chen GQ: Protein kinase cdelta mediates retinoic acid and phorbol myristate acetate-induced phospholipid scramblase 1 gene expression: Its role in leukemic cell differentiation. Blood 2004;104:3731-3738.

17 Wei S, Gao X, Du J, Su J, Xu Z: Angiogenin enhances cell migration by regulating stress fiber assembly and focal adhesion dynamics. PLoS ONE 2011;6:e28797.

18 Basse F, Stout JG, Sims PJ, Wiedmer T: Isolation of an erythrocyte membrane protein that mediates $\mathrm{Ca}^{2+}$ dependent transbilayer movement of phospholipid. J Biol Chem 1996;271:17205-17210.

19 Zhou Q Zhao J, Stout JG, Luhm RA, Wiedmer T, Sims PJ: Molecular cloning of human plasma membrane phospholipid scramblase. A protein mediating transbilayer movement of plasma membrane phospholipids. J Biol Chem 1997;272:18240-18244. 
20 Sahu SK, Gummadi SN, Manoj N, Aradhyam GK: Phospholipid scramblases: An overview. Arch Biochem Biophys 2007;462:103-114.

21 Zhou Q Ben-Efraim I, Bigcas JL, Junqueira D, Wiedmer T, Sims PJ: Phospholipid scramblase 1 binds to the promoter region of the inositol 1,4,5-triphosphate receptor type 1 gene to enhance its expression. J Biol Chem 2005;280:35062-35068.

22 Xu ZP, Tsuji T, Riordan JF, Hu GF: Identification and characterization of an angiogenin-binding DNA sequence that stimulates luciferase reporter gene expression. Biochemistry 2003;42:121-128.

23 Xu ZP, Tsuji T, Riordan JF, Hu GF: The nuclear function of angiogenin in endothelial cells is related to rrna production. Biochem Biophys Res Commun 2002;294:287-292.

24 Kusano S, Eizuru Y: Human phospholipid scramblase 1 interacts with and regulates transactivation of htlv1 tax. Virology 2012;432:343-352.

25 Silverman RH, Halloum A, Zhou A, Dong B, Al-Zoghaibi F, Kushner D, Zhou Q Zhao J, Wiedmer T, Sims PJ: Suppression of ovarian carcinoma cell growth in vivo by the interferon-inducible plasma membrane protein, phospholipid scramblase 1. Cancer Res 2002;62:397-402.

26 Bateman A, Finn RD, Sims PJ, Wiedmer T, Biegert A, Soding J: Phospholipid scramblases and tubbylike proteins belong to a new superfamily of membrane tethered transcription factors. Bioinformatics 2009;25:159-162.

27 Kuo YB, Chan CC, Chang CA, Fan CW, Hung RP, Hung YS, Chen KT, Yu JS, Chang YS, Chan EC: Identification of phospholipid scramblase 1 as a biomarker and determination of its prognostic value for colorectal cancer. Mol Med 2011;17:41-47.

-28 Kodigepalli KM, Anur P, Spellman P, Sims PJ, Nanjundan M: Phospholipid scramblase 1, an interferonregulated gene located at 3q23, is regulated by snon/skil in ovarian cancer cells. Mol Cancer 2013;12:32. 